\title{
Mathematical Analysis of Environmental Effects of Forming a Platoon of Smart Vehicles
}

\author{
Young Dae Ko ${ }^{1, * \mathbb{C}}$, Byung Duk Song ${ }^{2}$ and Yonghui $\mathrm{Oh}^{3}$ \\ 1 Department of Hotel and Tourism Management, Sejong University, Seoul 05006, Korea \\ 2 School of Industrial Engineering, Purdue University; West Lafayette, IN 47907, USA; bdsong@purdue.edu \\ 3 Department of Industrial Engineering, Daejin University; Pocheon 11159, Korea; oryong@daejin.ac.kr \\ * Correspondence: youngdae.ko@sejong.ac.kr; Tel.: +82-2-6935-2490
}

Received: 6 December 2018; Accepted: 18 January 2019; Published: 22 January 2019

\begin{abstract}
The platooning of autonomous smart vehicles in a high-speed driving environment is a technology that can be achieved in the near future, and some similar driving-assistive technologies have already been put in practice. There are a variety of advantages to platooning, and one of them is the environmental aspect. This study endeavored to perform a mathematical analysis of the environmental aspect of forming a platoon of smart vehicles. For that, a mathematical experiment was designed with four kinds of scenarios, and each scenario had a different operation profile in terms of the velocity and acceleration of each smart vehicle. The power consumption and both forming distance and time were derived variously according to each scenario through mathematical experiments, and it was observed that the length of the acceleration period and the average operation velocity of the smart vehicles had a significant impact on the environmental effect of platoon formation. It is hoped that this study will be a touchstone for various kinds of platooning algorithm research considering the environment.
\end{abstract}

Keywords: environmental effect; smart vehicle; platooning; power modeling; power consumption

\section{Introduction}

With the development of information and communications technology, various sensors have been installed in vehicles, making it possible to collect a great amount information in real time while the vehicles are running or stopped. In addition, using wireless internet technology, it is also possible to exchange information between vehicles (V2V) or between vehicles and infrastructure (V2I). These vehicles are called smart vehicles and are part of the innovative evolution of vehicles in the automotive industry [1]. In this way, the plurality of smart vehicles can exchange information on each other's speed, acceleration, and relative position and can collect traffic information, driving information of surrounding vehicles, and other environmental information, such as the weather, temperature, and pedestrians, through various sensors. Using such information, autonomous driving, in which the driver operates the vehicle automatically without driving the vehicle directly, has recently become the biggest concern [2].

Ultimately, it is regarded that smart vehicles will always be able to drive autonomously, but the most salient technology at this time is platooning in a high-speed driving environment, as shown in Figure 1 [3]. In a high-speed driving environment, most vehicles are operated at a velocity close to the maximum permissible speed, except in the occurrence of traffic congestion. In addition, the movement of the vehicles is monotonous because, in general, it is assumed that the operating situation is on an expressway or highway. In order to perform a platooning of smart vehicles, the speed, acceleration, and relative position data of each vehicle should be collected and utilized through vehicle-to-vehicle 
and vehicle-to-infrastructure communication. It is expected that the application of platooning in a high-speed driving environment will provide significant convenience for drivers.

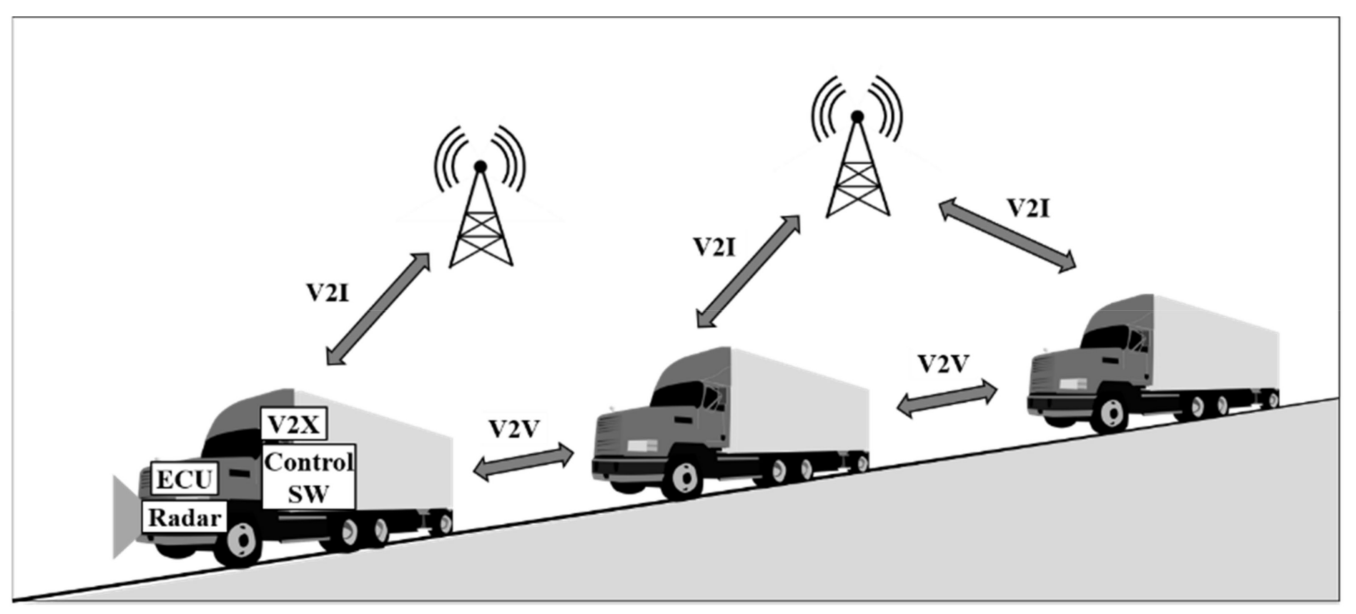

Figure 1. Concept of platooning.

Beyond the convenience for the drivers, there are several additional advantages, such as fuel reduction and congestion relaxation, when a series of smart vehicles are platooned. Then, as a result, the quantity of air pollution emissions can be reduced [3,4]. However, the effects of those advantages can diminish when there is insufficient consideration of the environmental aspect of how to form, maintain, and terminate a platoon.

Until now, most research on this topic has focused on technical issues, such as various communication and sensor technologies that enable smart vehicles to platoon. However, for successful practical application, platooning management issues should be dealt with to maximize the advantages of this technology. Therefore, this study sought to confirm the influence of forming a platoon on environmental pollution by calculating power consumption. Based on this research, in the future, it is hoped that various kinds of platooning algorithms for smart vehicles will be developed considering environmental pollution.

This paper is organized as follows. In Section 2, related previous studies are presented as the literature review. The power modeling used to calculate power consumption when smart vehicles form a platoon is described in Section 3, and the experimental design used to derive the power consumption of smart vehicles in several platooning situations is introduced in Section 4. In addition, the experimental results concerning environmental issues related to forming a platoon of smart vehicles are analyzed in Section 5. Finally, the findings and insights of this study are addressed in the conclusion in Section 6.

\section{Literature Review}

Though this paper does not discuss the technological aspect of smart vehicles, several studies are presented to develop the reader's understanding of the subject. In order to construct or to maintain a smart vehicle platoon, the communication technology used to exchange information between the smart vehicles is of vital importance. Through a V2V communication system, vehicles can transmit information such as vehicle speed. The authors of [5] dealt with V2V communication systems that allow vehicle configuration for platooning. There, they measured the performance of the first V2V system prototype. However, conventional wireless communication technologies such as IEEE 802.11p have performance issues related to heavy traffic conditions. Therefore, the authors of [6] studied the feasibility of a visible light communication (VLC) system for sharing information between vehicles when a vehicle is operating in a platoon and showed that if the VLC system is applied to the platoon, communication is possible even with optical noise. Building on this, a new communication protocol 
that combines radio and VLC systems to solve RF jamming attacks has been suggested. The authors of [7] performed simulations of a proposed protocol that can maintain a high message delivery rate despite the presence of RF jamming attacks. In addition to communication technology for sending and receiving information between vehicles, technology to identify and control vehicles is also important. The authors of [8] introduced a vision-based control for vehicle platooning. Based on the proposed visual tracking algorithm, a follower vehicle can track the leading vehicle directly using an onboard camera attached to the follower vehicle. There are several studies that have dealt with both communication and control technologies. The authors of [9] demonstrated cooperative driving with automated vehicles using the Differential Global Positioning System (DGPS) and intervehicle communication function dedicated short-range communication (DSRC). By demonstrating stop and go, platooning, merging, and obstacle avoidance functions, they confirmed the feasibility of communication and control technologies between vehicles.

In addition, there are several studies that investigated an operation method or algorithm for platooned smart vehicles. Most of these studies have investigated the operation methods to maintain platooning status. Those methods can be divided into centralized and decentralized approaches. For the centralized approach, the authors of [10] introduced a local controller that could adjust for speed to form a platoon with another heavy-duty vehicle (HDV) using the position, speed, and destination information of a vehicle. They verified the effect of the proposed algorithm through a simulation on the German autobahn road network. The authors of [11] studied the situation of platooned vehicles operating on an automated highway system (AHS). AHS uses computer control, communication, and sensing to increase road throughput and safety. They developed an algorithm for constructing a vehicle platoon at the entrance of the highway, which maximized the distance between vehicles at which the platoon could be maintained. For the decentralized approach, the authors of [12] introduced a platooning algorithm based on a near-to-near decentralized approach that allows a convoy to safely follow the leader's path without collisions or deviation from the platoon. There have been several studies that have mainly considered the construction of a platoon. The authors of [13] studied strategies of truck platooning. They analyzed how the behavior patterns of trucks vary according to the two strategies that make up the platoon (the catch-up and slow-down strategies). Also, they proposed a hybrid strategy that combines these two strategies and then compared the effects. The authors of [14] dealt with a situation in which vehicles construct a platoon when each vehicle has a deadline for the time of arrival at its destination. Fifty examples for the German road network were solved. Several small problems were solved using LINDO, and complex problems were solved using genetic algorithms. Though they investigated the formation of a smart vehicle platoon, they only introduced simple rule-based heuristics.

Moreover, there have been several studies of the environmental effects of smart vehicles when they form and operate as a platoon. In general, the environmental benefits are the result of aerodynamic drag reduction due to platooning. There have been several studies that have calculated such general environmental benefits. The author of [15] dealt with the operation of groups of HDVs. They modeled a two-layer control architecture for platooning and computed the fuel-optimal speed profile for HDV using dynamic programming within a proposed architecture. Simulations in various scenarios have shown that fuel savings of up to $12 \%$ can be achieved. The authors of [16] investigated the environmental and safety benefits of platooning systems. From an environmental point of view, they found through simulation that fuel consumption was reduced by $15 \%$ when smaller vehicles followed a heavy vehicle. Fuel savings has been adopted as a general criterion to calculate environmental benefits, and several studies investigated platooning situations using that criterion. The author of [17] suggested a framework for configuring HDV platoon formations, such as vehicle path change, departure time adjustment, and speed profile adjustment, the goal of which was fuel savings. Adjusting the speed of the vehicle through catch-up adjustment was the focus of this study. There, they investigated a proposed model with a simulation of the German road network, and it showed that the composition of the platoon can increase the fuel savings. The authors of [18] suggested 
the formation and break-up of a platoon to maximize fuel savings. They proposed an algorithm that can determine a truck's routing, velocity profile, etc., to form the platoon and verified their algorithm through examples. In addition, there have been a few studies that have provided several important insights on platooning situations considering environmental benefits. The authors of [4] analyzed the effect of fuel savings from HDV platooning. They found that with a shorter relative distance between vehicles, fuel savings can be maximized through air drag reduction and proper control.

Though there are previous studies that have dealt with the environmental aspect of platooned smart vehicles, few have investigated the environmental effect of smart vehicles trying to form a platoon at the microlevel. This study considered such a scenario by calculating power consumption, which has provided many important insights.

\section{Power Modeling}

When a vehicle is operating, there are five kinds of forces: rolling resistance $\left(F_{r r}\right)$, aerodynamic $\operatorname{drag}\left(F_{a d}\right)$, hill climbing $\left(F_{h c}\right)$, linear acceleration $\left(F_{l a}\right)$, and rotational acceleration, as shown in Figure 2. In general, the rotational acceleration force can be approximated to be $5 \%$ of the linear acceleration force [19]. The total required force $\left(F_{t e}\right)$ to operate a vehicle at time $t$ is shown in Equation (1):

$$
F_{t e}(t)=F_{r r}(t)+F_{a d}(t)+F_{h c}(t)+F_{l a}(t) .
$$

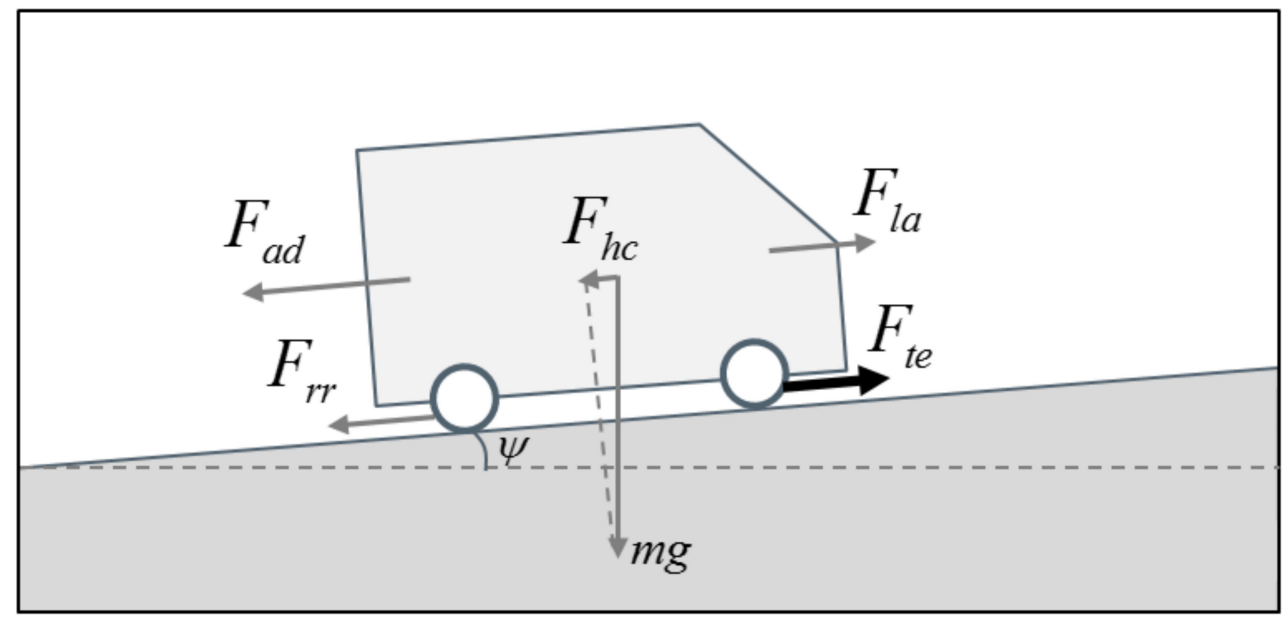

Figure 2. Five kinds of forces acting on a vehicle.

In addition, the total required power $\left(P_{t e}\right)$ at time $t$ can be calculated by multiplying both the total required force and the velocity at time $t$ (Equation (2)):

$$
P_{t e}(t)=F_{t e}(t) \cdot v(t)
$$

In detail, the total required power can be expressed with the acceleration and velocity at time $t$, as demonstrated in Equation (3), where $v(t)$ and $a(t)$ are the velocity and acceleration of a vehicle at time $t$, respectively. The remaining notations are denoted as follows; $\mu_{r r}$ is the coefficient of rolling resistance, $m$ is mass, $g$ is the acceleration of gravity, $\rho$ is air density, $A$ is the front area of the vehicle, $C_{d}$ is the coefficient of aerodynamic drag, and $\psi$ is the climbing angle [20]:

$$
P_{t e}(t)=\left\{\left(\mu_{r r} \cdot m \cdot g\right)+\left(\rho \cdot A \cdot C_{d} \cdot v(t) / 2\right)+(m \cdot g \cdot \sin \psi)+1.05(m \cdot a(t))\right\} \cdot v(t) .
$$

Note that, except for velocity and acceleration, all the elements are constant at time $t$, and regenerative braking is not considered in this model. 


\section{Mathematical Experiment Design}

\subsection{Basic Design}

In this study, the environmental effect of smart vehicle platoon formation was investigated through a mathematical-model-based analysis. For that, power modeling was performed (see Section 3), and a mathematical experiment was designed with several scenarios involving smart vehicle platoon formation.

To evaluate the power consumption of smart vehicles when they try to form a platoon, it was assumed that two smart vehicles were operating with at the same or different velocities, $v_{1}(t)$ and $v_{2}(t)$, and the same or different accelerations, $a_{1}(t)$ and $a_{2}(t)$, at time $t$. Also, they could control their velocities and accelerations adequately and differently at each time $t$. Finally, they could form a platoon with target platooning speed $v_{\text {target }}$ and target platooning space $d_{\text {safe }}$ within a predetermined forming time $t_{\text {forming, }}$ as shown in Figure 3.

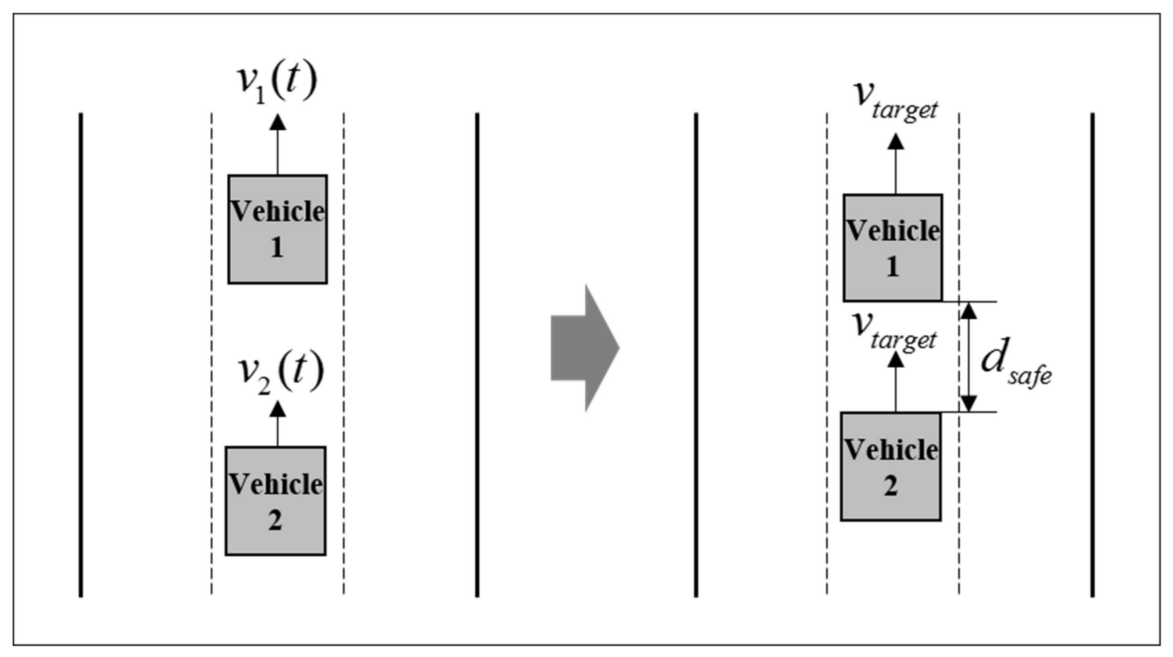

Figure 3. Experimental design of forming a platoon.

As observed in Equation (3), the power consumption of each smart vehicle at time $t$ can be differently calculated according to its velocity and acceleration at time $t$. Therefore, operation profiles of two smart vehicles should be predetermined exactly to derive their overall power consumption. Therefore, four kinds of scenarios about the different operation profile trends of two smart vehicles were assumed and analyzed in this study.

\subsection{Operation Profile Scenario}

It was assumed that there were two smart vehicles, smart vehicle 1 was running ahead of smart vehicle 2 , and the gap between the two smart vehicles was generally broader than the target platooning space, $d_{\text {safe }}$. Therefore, each smart vehicle should reduce the space between them by adequately controlling their velocity and acceleration. There are many possible operation profiles for this, and it is common that the velocity of smart vehicle 2 is faster than that of smart vehicle 1 . Based on this scheme, four kinds of operation profile scenarios were designed as follows:

- Scenario 1: firstly, smart vehicle 1 reduces its velocity to the minimum allowable level, while smart vehicle 2 increases its velocity to the maximum allowable level. Then, they control their velocities to reach the target platooning speed considering the final space between them to be the target platooning space.

- Scenario 2: firstly, smart vehicle 1 maintains its initial velocity, while smart vehicle 2 increases its velocity to the maximum allowable level. Then, they control their velocities to reach the target platooning speed considering the final space between them to be the target platooning space. 
- Scenario 3: firstly, smart vehicle 1 controls its velocity to reach the target platooning speed, while smart vehicle 2 increases its velocity to the maximum allowable level. Then, smart vehicle 2 reduces its velocity to reach the target platooning speed considering the final space between them to be the target platooning space.

- Scenario 4: firstly, smart vehicle 1 reduces its velocity to the minimum allowable level, while smart vehicle 2 controls its velocity as the target platooning speed. Then, smart vehicle 1 increases its velocity to reach the target platooning speed considering the final space between them to be the target platooning space.

Four kinds of scenarios for the operation profiles of two smart vehicles are depicted in Figure 4. It was assumed that the initial velocity of each smart vehicle was set as $v_{\text {initial }}$ and there was a maximum allowable velocity, $v_{\max }$, and a minimum allowable velocity, $v_{\min }$, while the target platooning speed was decided as $v_{\text {target }}$. There was different information about both the velocity and acceleration of each smart vehicle at every time $t$ according to each scenario.

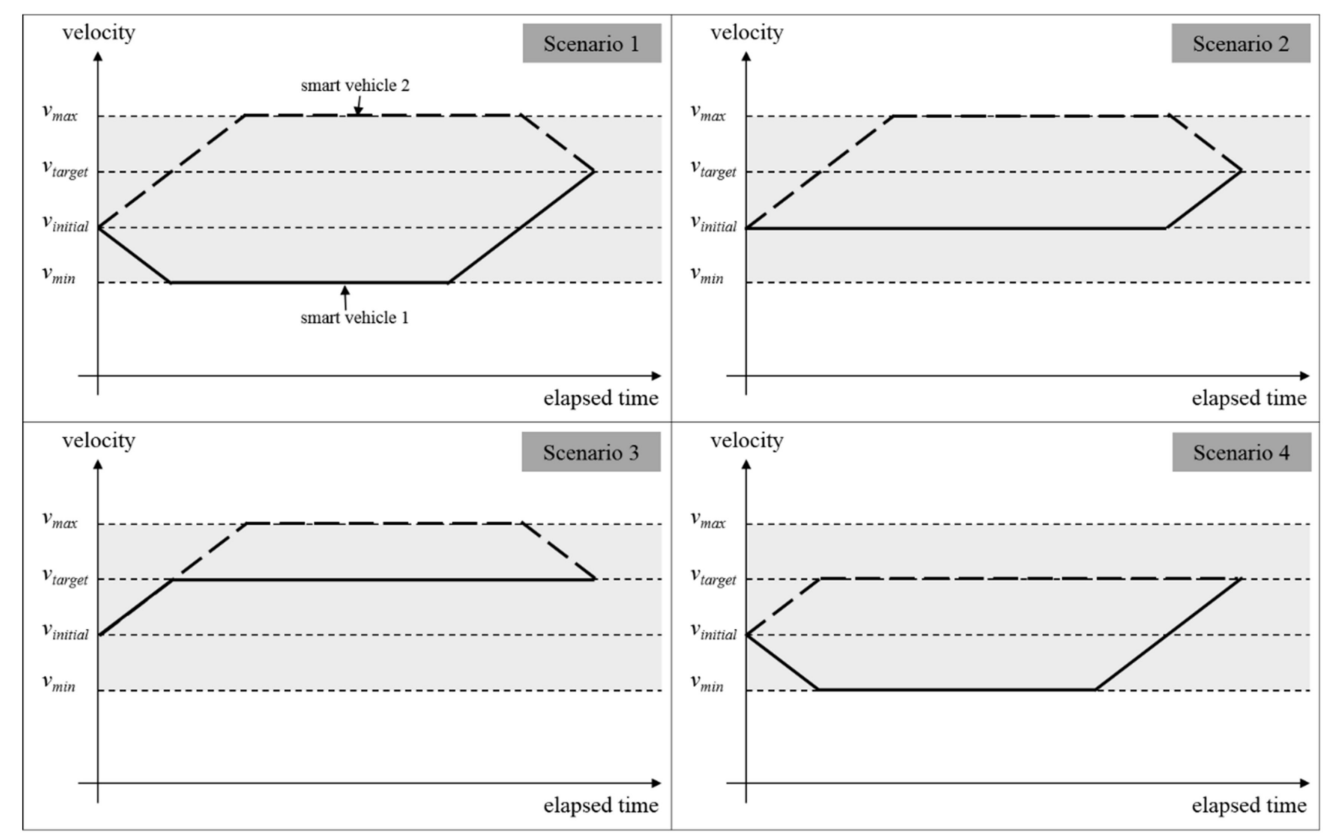

Figure 4. Operation profile of each scenario.

\section{Mathematical Experiment Result}

\subsection{Parameter Setting}

In this study, the mathematical experiments were designed to confirm the environmental effects of forming a platoon of smart vehicles with four kinds of operation profile scenarios. To perform the mathematical experiments, several parameters were set, as shown in Table 1.

Table 1. Parameters for the experiment.

\begin{tabular}{cccccc}
\hline Parameters & Values & Parameters & Values & Parameters & Values \\
\hline$v_{\text {initial }}$ & $81 \mathrm{~km} / \mathrm{h}$ & $t_{\text {forming }}$ & $60 \mathrm{~s}$ & $C_{d}$ & 1.2 \\
$v_{\text {target }}$ & $90 \mathrm{~km} / \mathrm{h}$ & $\mu_{r r}$ & 0.02 & $\psi$ & 0 \\
$v_{\text {min }}$ & $72 \mathrm{~km} / \mathrm{h}$ & $m$ & $2000 \mathrm{~kg}$ & $a_{1}$ & $0.25 \mathrm{~m} / \mathrm{s}^{2}$ \\
$v_{\text {max }}$ & $99 \mathrm{~km} / \mathrm{h}$ & $g$ & $9.8 \mathrm{~m} / \mathrm{s}^{2}$ & $a_{2}$ & $0.50 \mathrm{~m} / \mathrm{s}^{2}$ \\
$d_{\text {initial }}$ & $100 \mathrm{~m}$ & $\rho$ & 1 & $a_{3}$ & $1.25 \mathrm{~m} / \mathrm{s}^{2}$ \\
$d_{\text {safe }}$ & $5 \mathrm{~m}$ & $A$ & 5 & & \\
\hline
\end{tabular}


In this study, it was assumed that both smart vehicles initially started to form a platoon with the same velocity of $81 \mathrm{~km} / \mathrm{h}$, while the initial space between them was $100 \mathrm{~m}$ for all scenarios. After that, each smart vehicle operated separately according to the operation profile of each scenario within the minimum allowable velocity, $72 \mathrm{~km} / \mathrm{h}$, and the maximum allowable velocity, $99 \mathrm{~km} / \mathrm{h}$. Here, the smart vehicles should form a platoon upon reaching the target platooning speed, $90 \mathrm{~km} / \mathrm{h}$, and the target platooning space, $5 \mathrm{~m}$, within a predetermined forming time, $60 \mathrm{~s}$. In addition, to evaluate the environmental effects of forming a platoon of smart vehicles with different accelerations, the smart vehicles were designed to operate with three different accelerations $-0.25,0.50$, and $1.25 \mathrm{~m} / \mathrm{s}^{2}$-in each scenario. Moreover, note that there were several parameters for applying the power modeling, such as the coefficient of rolling resistance $\left(\mu_{r r}\right)$, mass of the smart vehicle $(m)$, acceleration of gravity $(g)$, air density $(\rho)$, front area of the vehicle $(A)$, the coefficient of aerodynamic drag $\left(C_{d}\right)$, and climbing angle $(\psi)$.

\subsection{Results Analysis}

A mathematical experiment was performed using the power modeling presented in Section 3 under four different kinds of operation profile scenarios by assuming the situation that two smart vehicles were trying to form a platoon. The power consumption, forming time, and forming distance until forming a platoon were variously derived according to each scenario, as shown in Table 2 . In addition, the variation of those elements was observed with three different accelerations.

Table 2. Numerical results.

\begin{tabular}{|c|c|c|c|c|c|c|}
\hline & \multicolumn{3}{|c|}{ Scenario 1} & \multicolumn{3}{|c|}{ Scenario 2} \\
\hline & $0.25 \mathrm{~m} / \mathrm{s}^{2}$ & $0.50 \mathrm{~m} / \mathrm{s}^{2}$ & $1.25 \mathrm{~m} / \mathrm{s}^{2}$ & $0.25 \mathrm{~m} / \mathrm{s}^{2}$ & $0.50 \mathrm{~m} / \mathrm{s}^{2}$ & $1.25 \mathrm{~m} / \mathrm{s}^{2}$ \\
\hline Power consumption (W) & 572,004 & 566,168 & 558,035 & 509,928 & 492,028 & 481,237 \\
\hline $\begin{array}{c}\text { Forming } \\
\text { time (seconds) }\end{array}$ & 29.0 & 21.0 & 16.0 & 34.0 & 26.5 & 22.0 \\
\hline \multirow[t]{3}{*}{$\begin{array}{c}\text { Forming } \\
\text { distance (meters) }\end{array}$} & 641.25 & 450.00 & 331.25 & 776.25 & 601.25 & 496.25 \\
\hline & \multicolumn{3}{|c|}{ Scenario 3} & \multicolumn{3}{|c|}{ Scenario 4} \\
\hline & $0.25 \mathrm{~m} / \mathrm{s}^{2}$ & $0.50 \mathrm{~m} / \mathrm{s}^{2}$ & $1.25 \mathrm{~m} / \mathrm{s}^{2}$ & $0.25 \mathrm{~m} / \mathrm{s}^{2}$ & $0.50 \mathrm{~m} / \mathrm{s}^{2}$ & $1.25 \mathrm{~m} / \mathrm{s}^{2}$ \\
\hline Power consumption $(\mathrm{W})$ & 636,827 & 605,634 & 586,917 & 480,911 & 458,717 & 443,587 \\
\hline $\begin{array}{l}\text { Forming } \\
\text { time (seconds) }\end{array}$ & 58.0 & 48.0 & 42.0 & 34.0 & 26.5 & 22.0 \\
\hline $\begin{array}{c}\text { Forming } \\
\text { distance (meters) }\end{array}$ & 1436.25 & 1192.50 & 1046.25 & 741.25 & 560.00 & 451.25 \\
\hline
\end{tabular}

Regarding power consumption, the power consumption of scenario 4 derived the smallest value, $480,911 \mathrm{~W}$, among the four kinds of scenarios, while that of scenario 3 was calculated to be $636,827 \mathrm{~W}$. That is, the operation profile of scenario 4 was the eco-friendliest way to form a platoon among the four kinds of scenarios considered and it reduced power consumption by $24.48 \%$ compared with that of scenario 3.

This was caused by the effects of the different operation velocities and length of acceleration periods of each scenario. For the length of acceleration period, smart vehicle 1 operated with velocities from 72 to $90 \mathrm{~km} / \mathrm{h}$, while smart vehicle 2 drove with velocities from 81 to $90 \mathrm{~km} / \mathrm{h}$ in scenario 4 . However, smart vehicle 1 operated with velocities from 72 to $90 \mathrm{~km} / \mathrm{h}$ and smart vehicle 2 drove with velocities from 81 to $99 \mathrm{~km} / \mathrm{h}$ in scenario 1 . Therefore, the power consumption of scenario 1 was greater than that of scenario 4 . In both scenarios 2 and 3, one smart vehicle operated with velocities from 81 to $99 \mathrm{~km} / \mathrm{h}$ while the other smart vehicle drove with velocities from 81 to $90 \mathrm{~km} / \mathrm{h}$. Though the length of acceleration periods of both scenarios 2 and 3 were similar to that of scenario 4 , the average velocities of the two smart vehicles of both scenarios 2 and 3 were higher than that of scenario 4 . Therefore, the power consumption of both scenarios 2 and 3 was greater than that of scenario 4 because 
power could be generated by multiplying both force and velocity while force is a function of velocity and acceleration. For the same reason, the power consumption of scenario 3 was greater than that of scenario 2, even though they had the same length of acceleration period.

In terms of forming distance and forming time, those of scenario 1 had the smallest values among the four kinds of scenarios, while those of scenario 3 had the highest values. In addition, the forming time of both scenarios 2 and 4 were exactly the same, while the forming distance of scenario 2 was slightly higher than that of scenario 4 .

This is due to the difference in operation velocities between the two smart vehicles in each scenario. In scenario 1 , the maximum difference of operation velocities between the two smart vehicles was $27 \mathrm{~km} / \mathrm{h}$, while that of scenario 3 was just $9 \mathrm{~km} / \mathrm{h}$. That is, the two smart vehicles in scenario 1 could form a platoon more rapidly because they could reduce the space between them more quickly. Therefore, the forming distance and forming time of scenario 1 had the smallest values, while those of scenario 3 had the highest values. In both scenarios 2 and 4 , the maximum differences of operation velocities between the two smart vehicles were the same $(18 \mathrm{~km} / \mathrm{h})$. Therefore, the forming time of both scenarios 2 and 4 were the same. However, the forming distance of scenario 2 was slightly higher than that of scenario 4 because the average velocity of scenario 2 was higher than that of scenario 4 .

Lastly, regarding acceleration, all elements, such as power consumption, forming time, and distance, tended to decrease when the acceleration value increased. That is, the smart vehicles tried to operate at the maximum allowable acceleration when they formed a platoon to reduce the environmental impact and to decrease the required time to form a platoon. Note that the distance variation between the two smart vehicles at elapsed time $t$ according to each scenario can be depicted as in Figure 5.

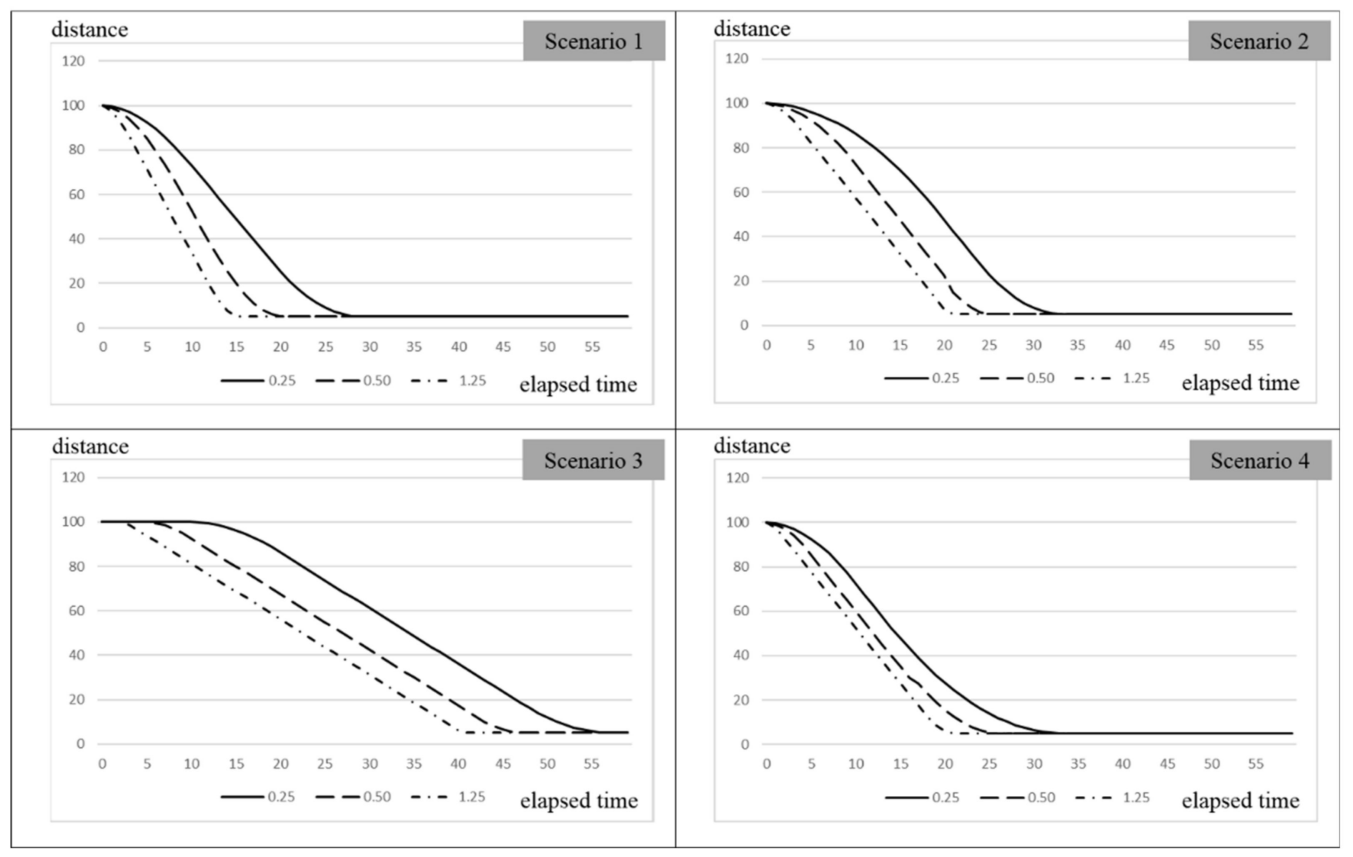

Figure 5. Distance variation between two smart vehicles of each scenario.

In this mathematical experiment, the climbing angle was set as zero. This is because the hill climbing force (Equation (3)) was not a function of either velocity or acceleration, and the average velocity, which was multiplied to derive the power, was similar in each scenario; therefore, it could not be influenced in each scenario differently. That is, some amount of power will be added or reduced according to the assumed value of the climbing angle. 


\section{Conclusions}

In this study, a mathematical analysis of the environmental effect of forming a platoon of smart vehicles was performed through a mathematical experiment and power modeling. Power consumption was adopted as an element for measuring the environmental effect when the smart vehicles formed a platoon and was calculated using the power modeling introduced in Section 3. Four kinds of different operation profile scenarios were designed by assuming a situation where two smart vehicles tried to form a platoon, and the mathematical experiment was executed with the parameters set by considering a high-speed driving environment.

Based on the results of the mathematical experiment, several meaningful insights are derived. Regarding power consumption, which represents the effect on the environment, both the length of acceleration period and the average operation velocity of smart vehicles have a significant impact. Since the positive acceleration of smart vehicles results in a large amount of power consumption, they should not use positive acceleration, if possible, when trying to form a platoon. In addition, assuming a high-speed driving environment, the average operation velocity of smart vehicles should be as low as possible when trying to form a platoon because power consumption is a function of force and velocity. Moreover, the average operation velocity of smart vehicles has no positive effect on reducing forming time and, in fact, has negative effects on the forming distance. From the perspective of both forming time and forming distance, only the difference of operation velocities between smart vehicles has a significant impact. Both forming time and distance can be reduced when there is a higher difference of operation velocities between smart vehicles. Therefore, smart vehicles should increase the difference of operation velocities between them to more rapidly form a platoon. Lastly, the high value of acceleration can reduce power consumption, forming time, and forming distance simultaneously. Therefore, smart vehicles should operate with the maximum allowable acceleration when they form a platoon to reduce the environment impact and to decrease the required time to form a platoon. Such insights are significantly important for developing and applying a simple rule-based algorithm for smart vehicles when they attempt to form a platoon.

For future research, we shall endeavor to develop an algorithm for the optimal operation profile for smart vehicles when trying to form a platoon considering environmental effects, and the present study will be essential to reach that end. In addition, it is hoped that various kinds of research related to the operation profile of smart vehicles with eco-friendly approaches can be performed by a variety of researchers.

Author Contributions: Y.D.K. defined the concept and topic of this study. Also, he designed, performed, and analyzed the mathematical experiments. B.D.S. and Y.O. investigated the previous studies and reviewed the overall manuscript.

Funding: This research was supported by the National Research Foundation of Korea (NRF) grant funded by the Korean government (MSIP; Ministry of Science, ICT \& Future Planning) (No. 2017R1C1B5017672).

Conflicts of Interest: The authors declare no conflict of interests.

\section{References}

1. Andersen, P.B.; Hauksson, E.B.; Pedersen, A.B.; Gantenbein, D.; Jansen, B.; Andersen, C.A.; Dall, J. Smart Grid Applications, Communications, and Security. In Smart Grid Applications, Communications, and Security; Wiley: Hoboken, NJ, USA, 2012; pp. 381-408.

2. Urmson, C.; Anhalt, J.; Bagnell, D.; Baker, C.; Bittner, R.; Clark, M.N.; Dolan, J.; Duggins, D.; Galatail, T.; Geyer, C.; et al. Autonomous driving in urban environments: Boss and the urban challenge. J. Field Robot. 2008, 25, 425-466. [CrossRef]

3. Saeednia, M.; Menendez, M. A consensus-based algorithm for truck platooning. IEEE Trans. Intell. Transp. Syst. 2017, 18, 404-415. [CrossRef]

4. Al Alam, A.; Gattami, A.; Johansson, K.H. An experimental study on the fuel reduction potential of heavy duty vehicle platooning. In Proceedings of the 13th International IEEE Conference on Intelligent Transportation Systems (ITSC), Funchal, Portugal, 19-22 September 2010; pp. 306-311. 
5. Bergenhem, C.; Hedin, E.; Skarin, D. Vehicle-to-vehicle communication for a platooning system. Procedia Soc. Behav. Sci. 2012, 48, 1222-1233. [CrossRef]

6. Abualhoul, M.Y.; Marouf, M.; Shagdar, O.; Nashashibi, F. Platooning control using visible light communications: A feasibility study. In Proceedings of the 16th International IEEE Conference on Intelligent Transportation Systems (ITSC), The Hague, The Netherlands, 6-9 October 2013; pp. 1535-1540.

7. Ishihara, S.; Rabsatt, R.V.; Gerla, M. Improving reliability of platooning control messages using radio and visible light hybrid communication. In Proceedings of the 2015 IEEE Vehicular Networking Conference (VNC), Kyoto, Japan, 16-18 December 2015; pp. 96-103.

8. Benhimane, S.; Malis, E.; Rives, P.; Azinheira, J.R. Vision-based control for car platooning using homography decomposition. In Proceedings of the 2005 IEEE International Conference on Robotics and Automation, Barcelona, Spain, 18-22 April 2005; pp. 2161-2166.

9. Kato, S.; Tsugawa, S.; Tokuda, K.; Matsui, T.; Fujii, H. Vehicle control algorithms for cooperative driving with automated vehicles and intervehicle communications. IEEE Trans. Intell. Transp. Syst. 2002, 3, 155-161. [CrossRef]

10. Larson, J.; Kammer, C.; Liang, K.Y.; Johansson, K.H. Coordinated route optimization for heavy-duty vehicle platoons. In Proceedings of the 16th International IEEE Conference on Intelligent Transportation Systems (ITSC), The Hague, The Netherlands, 6-9 October 2013; pp. 1196-1202.

11. Hall, R.; Chin, C. Vehicle sorting for platoon formation: Impacts on highway entry and throughput. Transport. Res. Part C Emer. Technol. 2005, 13, 405-420. [CrossRef]

12. Yazbeck, J.; Scheuer, A.; Charpillet, F. Decentralized near-to-near approach for vehicle platooning based on memorization and heuristic search. In Proceedings of the 2014 IEEE International Conference on Robotics and Automation (ICRA), Hong Kong, China, 31 May-7 June 2014; pp. 631-638.

13. Saeednia, M.; Menendez, M. Analysis of Strategies for Truck Platooning: Hybrid Strategy. Transp. Res. Rec. J. Transp. Res. Board 2016, 2547, 41-48. [CrossRef]

14. Nourmohammadzadeh, A.; Hartmann, S. The fuel-efficient platooning of heavy duty vehicles by mathematical programming and genetic algorithm. In Proceedings of the International Conference on Theory and Practice of Natural Computing, Sendai, Japan, 12-13 December 2016; pp. 46-57.

15. Turri, V.; Besselink, B.; Johansson, K.H. Cooperative look-ahead control for fuel-efficient and safe heavy-duty vehicle platooning. IEEE Trans. Control Syst Technol. 2017, 25, 12-28. [CrossRef]

16. Davila, A.; Nombela, M. Platooning-Safe and Eco-Friendly Mobility; SAE Technical Paper No. 2012-01-0488; SAE Int.: Warrendale, PA, USA, 2012.

17. Liang, K.Y. Coordination and Routing for Fuel-Efficient Heavy-Duty Vehicle Platoon Formation; KTH Royal Institute of Technology: Stockholm, Sweden, 2014.

18. Van De Hoef, S.; Johansson, K.H.; Dimarogonas, D.V. Fuel-optimal centralized coordination of truck platooning based on shortest paths. In Proceedings of the 2015 American Control Conference (ACC), Chicago, IL, USA, 1-3 July 2015; pp. 3740-3745.

19. Ko, Y.D.; Jang, Y.J. The optimal system design of the online electric vehicle utilizing wireless power transmission technology. IEEE Trans. Intell. Transp. Syst. 2013, 14, 1255-1265. [CrossRef]

20. Larminie, J.; Lowry, J. Electric Vehicle Technology Explained; John Wiley and Sons: Hoboken, NJ, USA, 2004.

(C) 2019 by the authors. Licensee MDPI, Basel, Switzerland. This article is an open access article distributed under the terms and conditions of the Creative Commons Attribution (CC BY) license (http://creativecommons.org/licenses/by/4.0/). 